doi:10.1016/j.vaccine.2006.07.008

Copyright (c) 2006 Elsevier Ltd All rights reserved.

\title{
Performance of the Brighton collaboration case definition for hypotonic-hyporesponsive episode (HHE) on reported collapse reactions following infant vaccinations in the Netherlands
}

\author{
Patricia E. Vermeer-de Bondt ${ }^{a, *}$, Aida Džaferagić ${ }^{a}$, Silke David ${ }^{a}$ and \\ Nicoline A.T. van der Maas ${ }^{a}$
}

${ }^{a}$ Safety Surveillance and Consultation of the National Vaccination Programme, Centre for Infectious Disease Control, RIVM National Institute for Public Health and Environment, PO Box 1, 3720BA Bilthoven, The Netherlands

Received 3 April 2006; revised 29 June 2006; accepted 5 July 2006. Available online 24 July 2006.

Corresponding author. Tel.: +3130274 2424; fax: +3130274 4430 .

\begin{abstract}
We reviewed collapse (sudden onset of pallor, limpness and hyporesponsiveness) following the first infant (DPTP + Hib) vaccination reported to the enhanced passive surveillance system of the Netherlands in 1994-2003. All 1303 reports identified by the current RIVM (National Institute for Public Health and Environment) case definition were captured by the Brighton Collaboration (BC) case definition, with in $17(1.3 \%)$ reports insufficient information. Over the years the proportion of the highest level of diagnostic certainty (level 1) increased due to more complete data from $70 \%$ to over $90 \%$. We checked the BC case definition also on a sample of cases (with pallor or hyporesponsiveness) not meeting RIVM's case definition for collapse at the time. Sixty out of 200 cases were captured by BC but again rejected by RIVM. The sensitivity BC levels 2 and 3 appeared too high. We recommend a more restrict case definition by the Brighton Collaboration with certain exclusion criteria to make it more specific. Furthermore a change in the specifications for levels 2 and 3 will increase specificity and accommodate for the loss of sensitivity.
\end{abstract}

Keywords: Case definition; Collapse reaction; Adverse event following immunisation

\section{Introduction}

Collapse or hypotonic-hyporesponsive episode (HHE) is an acknowledged adverse reaction following infant vaccinations [1], [2], [3], [4] and [5]. Its core symptoms are sudden onset of pallor, limpness and hyporesponsiveness. Most reports concern vaccines containing pertussis. Collapse is also known to occur after single $\mathrm{Hib}$, hepatitis B, DTpolio or without vaccinations. Although acellular pertussis 
vaccines appear to have a lower rate than whole cell pertussis vaccines, collapse does still occur [6], [7], [8] and [9]. The pathogenesis of collapse following infant vaccinations is not yet fully understood and further study is needed [8]. Interand intra-study consistency of case definitions for collapse would be helpful. Not only differences in case definitions but also differences in schedules, (combination of) vaccines, methods of validation and complementation of information affect the comparability of vaccine safety data [4], [10] and [11]. The Brighton Collaboration (BC) aims to improve this situation by developing standardised case definitions and (reporting) guidelines for vaccine safety studies to be used in different health care settings. The case definition for collapse was among the first six published [12], [13], [14] and [15].

In the Netherlands, the National Institute for Public Health and Environment (RIVM) has used a single case definition over the past 15 years [4], [13] and [14]. In a current study of the increased rate of reported collapse reactions since the implementation of the accelerated vaccination schedule in 1999 we tested the consistency of its use. We also applied the BC case definition. Here we present the evaluation of the use of the $\mathrm{BC}$ case definition on reported collapse reactions.

\section{Methods}

RIVM has been monitoring the safety of the National Vaccination Programme of the Netherlands since 1962 . The backbone of the safety surveillance is an enhanced passive surveillance system. Adverse events following immunisations (AEFI) are also studied through systematic follow-up, questionnaires or other data sources. The vaccination schedule has been changed in 1999 with the first dose at 2 months instead of 3 months of age (Table 1 ).

Table 1.

Vaccination schedule of the Netherlands with whole cell pertussis vaccine; before 1999 start at 3 months; HepB only for certain risk groups

\begin{tabular}{|l|l|l|}
\hline Age at vaccination & Vaccines & \\
\hline 2 months & DPTP + Hib1 & HepB1 \\
\hline 3 months & DPTP + Hib2 & HepB2 \\
\hline 4 months & DPTP + Hib3 & \\
\hline 11 months & DPTP + Hib4 & HepB3 \\
\hline 14 months & MenC & MMR1 \\
\hline 4 years & DTPolio5 & aP \\
\hline 9 years & DTPolio6 & MMR2 \\
\hline
\end{tabular}

MenC was included in 2002 and combination vaccine DPTP-Hib in 2003. 


\subsection{Passive surveillance system}

Reported adverse events are assessed after validation and collection of complementary data. For potential collapse reactions, RIVM has increased its history taking from parents, from $60 \%$ to over $90 \%$ of cases. Collapse is most frequent after the first vaccinations and rare after the fourth dose at 1 year of age. The rate of reported collapse reactions has been rather stable until 1999 with a step up after implementation of the accelerated schedule with start at 2 months of age (Table 2). Since 1994 RIVM publishes all reported adverse events irrespective of causality [16, http://www.rivm.nl/].

Table 2.

Reported collapse reactions after DPTP + Hib vaccinations in the Netherlands

\begin{tabular}{|l|l|l|l|l|}
\hline Year of report $^{\text {a }}$ & \multicolumn{4}{|l|}{ Vaccine dose } \\
\hline & dh1 & dh2 & dh3 & dh4 \\
\hline 1994 & 98 & 13 & 5 & 4 \\
\hline 1995 & 96 & 23 & 9 & 2 \\
\hline 1996 & 85 & 17 & 16 & 3 \\
\hline 1997 & 106 & 23 & 10 & 5 \\
\hline 1998 & 103 & 28 & 20 & 5 \\
\hline 1999 & 147 & 43 & 27 & 4 \\
\hline 2000 & 151 & 41 & 21 & 8 \\
\hline 2001 & 173 & 59 & 29 & 7 \\
\hline 2002 & 198 & 48 & 20 & 4 \\
\hline 2003 & 146 & 39 & 19 & 6 \\
\hline & 1303 & 334 & 176 & 48 \\
\hline Total & \multicolumn{5}{|l}{} \\
\hline
\end{tabular}

a Since 1999 accelerated schedule with vaccination at 2, 3, 4 and 11 months of age.

${ }^{b} \mathrm{dh}=\mathrm{dptp}+$ hib vaccine (dose), all with whole cell pertussis component. 


\subsection{Case definitions for collapse (HHE)}

\subsubsection{Case definition with levels for completeness and exclusion criteria- RIVM}

(1) Sudden onset of triad of (complete or incomplete)

- Pallor and/or cyanosis

- Limpness

-Inclusion of absent limpness if chills or myoclonic jerks or hypertonicity are present (exceptionally); this is considered atypical collapse.

- Loss of consciousness

aExclusion of episodes without pallor or cyanosis.

aExclusion of episodes without marked reduction in responsiveness.

-Exclusion of fainting in older children and of clear cut breath holding spells with no pale phase and if any only very short loss of responsiveness.

Exclusion follows also if the symptoms are part of another diagnosis, i.e. postictal state, intoxication, septicaemia, sleep, death, etceteras.

\subsubsection{Case definition with levels of diagnostic certainty-Brighton Collaboration (BC)}

(1) Level 1 of diagnostic certainty

- Sudden onset of

„Limpness

-Reduced responsiveness

¿Pallor or cyanosis

(2) Level 2 of diagnostic certainty

- Sudden onset of

uTwo of the three symptoms of level 1 with the third unknown.

(3) Level 3 of diagnostic certainty

- Sudden onset of

uTwo of the three symptoms of level 1 with the third known to be absent.

(4) Level 4 
- Event not meeting case definition

uReported collapse with insufficient evidence to meet the case definition.

\subsection{Evaluation of cases and analysis}

1. All identified collapse reactions after the first infant vaccine dose reported in 1994-2003 were included. These have been considered collapse reaction at the time, with for marginal cases inclusion by consensus of RIVM researchers (PV and $A D$ or NM). We coded all cases again for (the degree of) pallor, cyanosis, limpness and hyporesponsiveness, and also for all other reported symptoms. We assessed these reports once more with the RIVM case definition and checked for consistency. Subsequently we applied the BC case definition to the same data.

2. We selected cases with pallor or cyanosis, apathy, drowsiness or limpness as working diagnosis or most prominent symptom reported in 2000-2003. These reports did not meet the RIVM case definition of collapse at the time. Now we applied both RIVM and BC case definitions on these reports.

Below we discuss the feasibility, sensitivity and specificity of the BC case definition on the datasets.

\section{Results}

\subsection{Collapse (HHE)}

The vast majority of the 1303 reported collapse reactions followed DPTP + Hib, 12 with simultaneous HepB vaccine; 12 followed DPTP only, one DTPolio and Hib. Most children received the vaccines in two injections, 103 in a single injection and one child in three injections. Five hundred and twenty-one cases followed vaccinations under the old schedule with start at 3 months and 782 under the new accelerated schedule (Fig. 1). This resulted in a rate of 5.1 per $10,000(95 \%$ CI: 4.5-5.7) and 8.6 per 10,000 first doses, respectively, according to data from the Dutch vaccination registers. There was a slight overrepresentation of boys $(707 ; 54 \%)$.

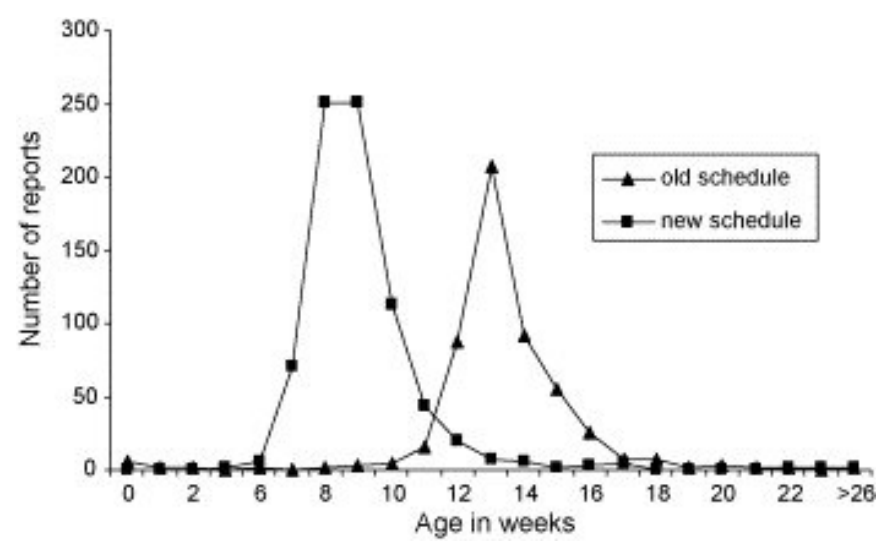

Fig. 1. Age distribution in weeks for collapse following the first vaccine dose, under the old schedule and the accelerated schedule of the Netherlands, reported in 1994-2003. 
According to the RIVM case definition, 660 (51\%) (probably) had complete collapse reaction with extreme pallor or cyanosis, loss of muscle tone and no reaction at all. In 403 cases (31\%), one or more of the symptoms were present to a lesser degree. $180(14 \%)$ lacked information on one of the core symptoms. A small percentage $(43 ; 3.3 \%)$ had one of the core symptoms reported as absent. Five cases with only slightly reduced muscle tone and little loss of responsiveness were considered borderline and 12 lacked specific information. These cases were considered to be possible collapse by RIVM at the time. Now they were reviewed and again accepted; at the time we were unable to get an eyewitness account. It must be noted that exclusion criteria are part of the RIVM case definition and it also takes into account the full clinical presentation.

All RIVM cases were identified by the BC case definition (Table 3). 1063 cases were captured by level 1 and 180 by level 2, representing $96 \%$ of the total. Forty-three $(3.5 \%)$ were level 3 and 17 did not meet the case definition (Table 4). By design all cases under level 1 were included in level 2. The proportion of level 1 increased somewhat over the years probably due to more complete information (Fig. 2).

Table 3.

Case definitions of collapse by RIVM and BC applied to cases after the first DPTP + Hib vaccinations reported in 1994-2003

\begin{tabular}{|l|l|l|l|l|l|}
\hline RIVM & \multicolumn{3}{l}{ BC } & \multicolumn{4}{l|}{} \\
\hline & $\begin{array}{l}\text { Level } \\
\mathbf{1}\end{array}$ & $\begin{array}{l}\text { Level } \\
\mathbf{2}\end{array}$ & $\begin{array}{l}\text { Level } \\
\mathbf{3}\end{array}$ & $\begin{array}{l}\text { Level } \\
\mathbf{4}\end{array}$ & Total \\
\hline Full (possibly) & 660 & & & & 660 \\
\hline Partial & 403 & & & & 403 \\
\hline Probably incomplete or atypical & & 180 & & & 180 \\
\hline Incomplete & & & 43 & & 43 \\
\hline Very incomplete, marginal or borderline & & & & 5 & 5 \\
\hline Not further specified & & & & 12 & 12 \\
\hline & & & & & 1303 \\
\hline Total & 1063 & 180 & 43 & 17 & 13 \\
\hline
\end{tabular}


Vaccine Volume 24, Issues 49-50, 30 November 2006, Pages 7066-7070

Table 4.

Presenting core symptoms in assessed collapse cases reported in 1994-2003

\begin{tabular}{|c|c|c|c|c|c|c|c|}
\hline \multirow{2}{*}{$\begin{array}{l}\text { BC } \\
\text { levels }\end{array}$} & \multirow[t]{2}{*}{ Total } & \multirow[t]{2}{*}{ Core symptoms } & \multirow[b]{2}{*}{ extreme } & \multirow[b]{2}{*}{ partial } & \multirow[b]{2}{*}{ present } & \multirow[b]{2}{*}{ absent } & \multirow[b]{2}{*}{$\begin{array}{l}\text { unknown } \\
\text { or not } \\
\text { recorded }\end{array}$} \\
\hline & & & & & & & \\
\hline \multirow[t]{3}{*}{ Level 1} & 1063 & Pallor & 1040 & 23 & 1063 & 0 & 0 \\
\hline & & Limpness & 888 & 175 & 1063 & 0 & 0 \\
\hline & & Unresponsiveness & 731 & 332 & 1063 & 0 & 0 \\
\hline \multirow[t]{3}{*}{ Level 2} & 180 & Pallor & 169 & 6 & 173 & 0 & 7 \\
\hline & & Limpness & 53 & 10 & 63 & 0 & 117 \\
\hline & & Unresponsiveness & 77 & 45 & 122 & 0 & 58 \\
\hline \multirow[t]{3}{*}{ Level 3} & 43 & Pallor & 45 & 0 & 45 & 0 & 1 \\
\hline & & Limpness & 8 & 2 & 10 & 36 & 0 \\
\hline & & Unresponsiveness & 17 & 16 & 33 & 13 & 0 \\
\hline \multirow[t]{3}{*}{ Level 4} & 17 & Pallor & 10 & 0 & 10 & 0 & 7 \\
\hline & & Limpness & 1 & 0 & 1 & 1 & 15 \\
\hline & & Unresponsiveness & 0 & 0 & 0 & 3 & 14 \\
\hline
\end{tabular}




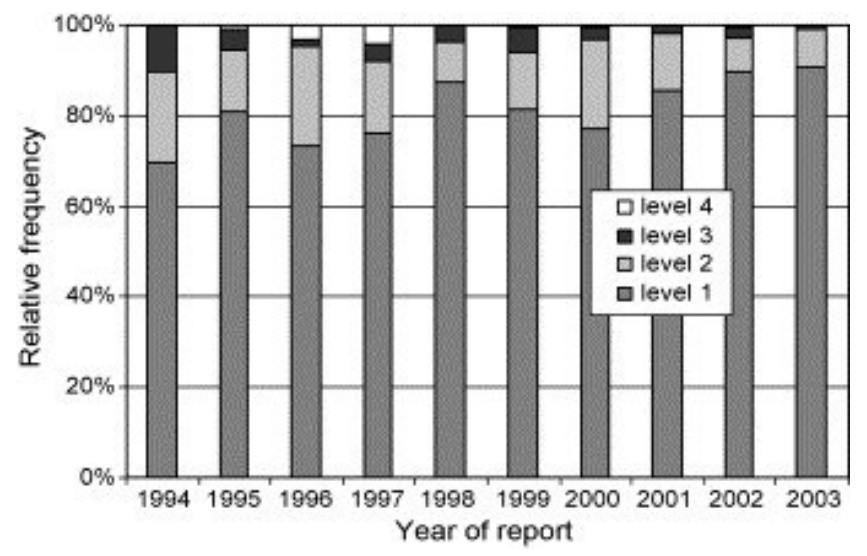

Fig. 2. Distribution of levels according to BC case definition of collapse (HHE) after the first infant vaccine dose in the Netherlands.

\subsection{Pallor and/or apathy}

The rejected marginal cases and cases with pallor, cyanosis, apathy or drowsiness as working diagnosis, reported in 2000-2003 (there were no cases with lameness, flaccidity or limpness as working diagnosis) were coded on relevant reported symptoms and their timing. For the RIVM case definition at least some degree of pallor or cyanosis must be present. Full conscience precludes the diagnosis of collapse also. Some cases had reported symptoms lasting for several days or weeks, not consistent with "episode". Those cases were excluded, as well as cases where the symptoms were part of another clinical entity/diagnosis. Cases with pallor in combination with drowsiness, reduced alertness, listlessness, lethargy or apathy were most problematic to assess both under RIVM and BC case definitions. The BC case definition captured 60 of the 200 cases rejected by RIVM (Table 5). This results in specificity for the BC case definition of $70 \%(95 \%$ CI: $64-76 \%$ ). Sensitivity of the BC definition based on (potential) collapse cases for those last four reporting years is $99.4 \%$ (95\% CI: $98.5-99.8 \%$ ), including four reports of BC level 4 (Table 6 ). For all 1303 cases sensitivity is $98.7 \%$ ( $95 \%$ CI: $97.9-99.2 \%)$. 
Vaccine Volume 24, Issues 49-50, 30 November 2006, Pages 7066-7070 
Vaccine Volume 24, Issues 49-50, 30 November 2006, Pages 7066-7070

Table 5.

RIVM grounds for exclusion of 60 cases with pallor and/or hyporesponsiveness captured by BC case definition

\begin{tabular}{|c|c|c|c|c|}
\hline \multirow[t]{2}{*}{ Exclusion grounds } & \multicolumn{4}{|c|}{ BC case definition level } \\
\hline & 1 & 2 & 3 & Total \\
\hline Pallor during normal sleep & 2 & 7 & 14 & 23 \\
\hline Sleepy, listless, lethargic, less alert, drowsy & 0 & 2 & 11 & 13 \\
\hline Crying throughout the episode & 3 & 0 & 2 & 5 \\
\hline Duration inconsistent with episode & 0 & 0 & 3 & 3 \\
\hline Other diagnosis, i.e. intoxication, severe airway infection, contaminated infant formula, BHS & 1 & 0 & 3 & 4 \\
\hline Too little to be called collapse & 3 & 3 & 1 & 7 \\
\hline Normal conscience or normal colour & 0 & 0 & 5 & 5 \\
\hline Total & 9 & 12 & 39 & 60 \\
\hline
\end{tabular}


Vaccine Volume 24, Issues 49-50, 30 November 2006, Pages 7066-7070 
Table 6.

Collapse according to RIVM and BC case definitions for reports in 2000-2003

\begin{tabular}{|l|l|l|l|}
\hline BC & \multicolumn{3}{l|}{ RIVM } \\
\hline & Yes & No & Total \\
\hline Yes & 664 & 60 & 724 \\
\hline No & 4 & 140 & 144 \\
\hline \multicolumn{4}{|l}{} \\
\hline Total & 668 & 200 & 868 \\
\hline
\end{tabular}

\section{Discussion}

We applied the $B C$ case definition on 1303 consecutive reported collapse reactions following the first vaccinations identified by the RIVM case definition. All were captured by the BC case definition apart from 17 cases of level 4 . This is not surprising since the two case definitions do not differ much. RIVM has tried to specify required minimal levels of the core symptoms. We consider (some) pallor and/or cyanosis an essential condition for collapse and also a minimal degree of hyporesponsiveness. Some other diagnoses are considered exclusion criteria.

The level of consciousness is most difficult to establish, not so much in case of total unresponsiveness as with possible hyporesponsiveness. Drowsy, listless, sleepy, sluggish, weary, dozy, somnolent, slumberous, dazed, passive, apathetic and similar terms are reported. Professionals or parents may use different terms for the same degree of symptoms or the same terms for different levels. In detailed history taking by RIVM this may be worked out but in reports without such additional information, this is often impossible to assess. The term hyporesponsiveness has no (case) definition or specifications. The term HHE is a misnomer because it does not include pallor, thus generating reports without (mentioned) paleness.

We feel that the $B C$ case definition is too sensitive for our validated cases in the passive system capturing 60 out of 200 false positives. If level 2 is adjusted by only allowing limpness to be unknown and requiring pallor/cyanosis and hyporesponsiveness to be present and level 3 harbours unknown pallor or unknown responsiveness, the definition gains in specificity. Also other exclusion criteria are recommended. In our current evaluation this would result in a loss of 13 cases in the collapse case series $(1303 ; 1 \%)$. On the other hand, it would prevent approximately 140 false positive cases among pallor/apathy reports in the passive system over the same period. The current level 3 might be used as capture diagnosis of cases further to be evaluated.

The high sensitivity may not pose a problem in clinical trials, or other withinstudy comparisons as long as all study arms are treated equally. For betweenstudy comparison this low specificity poses a problem. For the case series in our enhanced passive surveillance system with high prevalence of collapse ( $77 \%$ for 2000-2003), specificity is acceptable leading to overestimation of $10 \%$. If 
however the diagnosis of collapse has adverse consequences, like withholding further vaccinations, this may not be acceptable. In the Netherlands collapse is not regarded a contraindication.

For data sources with a lower prevalence of collapse, specificity could drop to $10 \%$ or lower with subsequent large overestimation of incidence rates (and consequences).

In short, application of the Brighton Collaboration case definitions of collapse was feasible in our case series. Adjustment is needed however. We warn against having too many exclusion criteria, and recommend only those that preclude collapse, i.e. normal level of consciousness, absent pallor and definite other diagnoses. Accompanying symptoms like apnoea, hives, discoloured legs, vomiting, jerks, chills or hypertonicity do not preclude collapse. These symptoms may be taken into account in stratified analysis and may (help to) elucidate possible pathogenesis. We strongly encourage the development and use of case definitions for adverse events following immunisation. Feedback on experience with it may lead to further improvement of the "fit" and applicability.

\section{References}

[1] Hannik CA, Cohen H. Pertussis vaccine experience in the Netherlands. Proceedings of the third international symposium on pertussis. Washington: DHEW Publications; 1979. p. 79-1830, 279-82.

[2] C.L. Cody, L.J. Baraff, J.D. Cherry, S.M. Marcy and C.R. Manclark, Nature and rates of adverse reactions with DTP and DT immunizations in infants and children, Pediatrics 68 (1981), pp. 650-660.

[3] Institute of Medicine. Adverse effects of pertussis and rubella vaccines: a report of the committee to review the adverse consequences of pertussis and rubella vaccines. Howson $\mathrm{CP}$, Howe $\mathrm{CJ}$, Fineberg HV, editors. Washington, DC: National Academy Press; 1991.

[4] P.E. Vermeer-de Bondt, J. Labadie and H.C. Rümke, Rate of recurrent collapse after vaccination with whole cell pertussis vaccine: follow up study, Br Med J 316 (1998), pp. 902-903.

[5] T. Jefferson, M. Rudin and DiPietrantonj, Systematic review of the effects of pertussis vaccines in children, Vaccine 21 (2003), pp. 2003-2014.

[6] E. Miller, Collapse reactions after whole cell pertussis vaccination, $\mathrm{Br}$ Med J 316 (1998), p. 876. [7] H. Heijbel, M.C. Ciofi degli Atti and E. Harzer et al., Hypotonic hyporesponsive episodes in eight pertussis vaccine studies, Dev Biol Stand 89 (1997), pp. 101-103.

[8] R. Gold, D. Scheifele and S. Halperin et al., Hypotonic-hyporesponsive episodes in children hospitalized at 10 Canadian pediatric tertiary-care centers, 1991-1994, CCDR 23 (1997), pp. 73-76.

[9] T.S. DuVernoy, M.M. Braun and the VAERS Working Group, Hypotonic-hyporesponsive episodes reported to the vaccine adverse event reporting system (VAERS), 1996-1998, Pediatrics 106 (2000), p. e52.

[10] Vermeer-de Bondt PE, Wesselo C, Dzaferagic A, Phaff TAJ. Adverse events following immunisation under the National Vaccination Programme of the Netherlands. Number VII-Reports in 2000. Bilthoven: RIVM report 000001006; 2002.

[11] M.S. Gold, Hypotonic-hyporesponsive episodes following pertussis vaccination, Drug Safety 25 (2002), pp. 85-90. 
[12] K.S. Kohl, J. Bonhoeffer, R. Chen, P. Duclos, H. Heijbel and U. Heininger et al., The Brighton Collaboration: enhancing comparability of vaccine safety data, Pharmacoepidemiol Drug Safety 4 (2003), pp. 335-340.

[13] M.M. Braun, G. Terracciano, M.E. Salive, D.A. Blumberg, P.E. Vermeer-de Bondt and H. Heijbel et al., Report on a US public health service workshop on hypotonic-hyporesponsive episode (HHE) after pertussis vaccination, Pediatrics 102 (1998), p. e52.

[14] J. Bonhoeffer, M.S. Gold, H. Heijbel, P. Vermeer, D. Blumberg and M. Braun et al., Hypotonichyporesponsive episode (HHE) as an adverse event following immunization: case definition and guidelines for data collection, analysis, and presentation, Vaccine 22 (2004), pp. 563-568.

[15] J. Bonhoeffer, K. Kohl, R. Chen, P. Duclos, H. Heijbel and U. Heiniger et al., The Brighton Collaboration: addressing the need for standardised case definitions of adverse events following immunization (AEFI), Vaccine 21 (2002), pp. 298-302.

[16] Vermeer-de Bondt PE, Džaferagić A, Phaff TAJ, Wesselo C, Maas van der NAT. Adverse events following immunisation under the National Vaccination Programme of the Netherlands; number XIreports in 2004. Bilthoven: RIVM Report 240071002, 2005. 